\title{
ANÁLISE DA CAPACIDADE COMPETITIVA DAS MICRO E PEQUENAS EMPRESAS BRASILEIRAS COM VISTA A EXPORTAÇÃO
}

\section{THE COMPETITIVE CAPACITY ANALYSIS OF MICRO AND SMALL BRAZILIAN COMPANIES WITH A VIEW TO EXPORT}

Recebido em: 31 jul. 2020

Aprovado em: 28 mar. 2021

Versão do autor aceita publicada online: 28 mar. 2021

Publicado online: 30 jun 2021

Como citar esse artigo - American Psychological Association (APA):

Ferreira, C. P. S., Cavalcanti, A. M., \& Silva, A. M. (2023, jan./mar.). Análise da capacidade competitiva das micro e pequenas empresas brasileiras com vista a exportação. Exacta, 21(1), 53-79. https://doi.org/10.5585/exactaep.2021.17813

Submeta seu artigo para este periódico $७$

Processo de Avaliação: Double Blind Review

Editor: ${ }^{D}$ Dr. Luiz Fernando Rodrigues Pinto

Dados Crossmark 



\title{
ANÁLISE DA CAPACIDADE COMPETITIVA DAS MICRO E PEQUENAS EMPRESAS BRASILEIRAS COM VISTA A EXPORTAÇÃO
}

\author{
THE COMPETITIVE CAPACITY ANALYSIS OF MICRO AND SMALL BRAZILIAN COMPANIES WITH A \\ VIEW TO EXPORT
}

\author{
(iD) Carla Patrícia Santos Ferreira ${ }^{1}$ \\ (iD) André Marques Cavalcanti² \\ (iD) Auristela Maria da Silva ${ }^{3}$
}

Resumo: As micro e pequenas empresas predominam em números frente as médias e grandes empresas na ordem de mais de 90\% em todo o mundo. Contribuindo fortemente na formação do Produto Interno Bruto em todos os países. Tornam-se propulsoras do desenvolvimento econômico na mediada em que recebem estímulos adequados em um ambiente que propicie o desenvolvimento de inovações tecnológicas. Observa-se na literatura que quando estabelecido o meio ambiente adequado, proporcionado por uma política pública de país, são desenvolvidos ganhos de capacidades competitivas nas empresas lá instaladas. Em contra partida, observa-se escassez na produção de artigos científicos que analisam como são estabelecidos ganhos da capacidade competitiva das empresas que participam de programas de subvenção e que se tornam capazes de se desenvolverem, tais quais, aquelas que participam de clusters de desenvolvimento tecnológico com o seu efetivo ganho de capacidade após a finalização da sua participação em programas de incubação. Nesse aspecto, o Brasil atua mais fortemente com subvenções que visam desenvolver a capacidade competitiva de empresas através de programa, tais como: Programa Nacional da Cultura Exportadora, Programa de Extensão Industrial para Exportação, Brasil Mais Produtivo, Agentes Locais de Inovação, entre outros. Tendo estes como finalidades a realização de consultorias e financiamentos para aquisição de equipamentos, além de consultorias para desenvolver as capacidades competitivas das empresas selecionadas de modo a atuarem em alto nível de competitividade. Assim, esta pesquisa visa analisar a capacidade competitiva voltada para a internacionalização de micro e pequenas empresas que participaram de programas de incentivos ao aumento de competitividade com vistas a identificar quais capacidades foram incluídas em suas estratégias de atuação, ao final do programa, que indique a sua efetividade. Para isso são analisados os relatórios de 256 empresas participantes do programa de Extensão Industrial para Exportação para identificar, pós execução do projeto, o nível de competitividade dessas empresas localizadas na região metropolitana da cidade do Recife.

Palavras-chave: pequenas e médias empresas; internacionalização; competitividade; inovação.

Abstract: Micro and small companies predominate in numbers compared to medium and large companies in the order of more than $90 \%$ worldwide. Contributing strongly to the formation of the Gross Domestic Product of all countries. They become drivers of economic development as they receive adequate stimuli in an environment that fosters the development of technological innovations in products and services. Observing in the literature that when the appropriate environment is established, provided by a public policy of the country, competitive capacity gains are developed in the companies installed there. On the other hand, there is a scarcity in the production of articles that seek to establish a correlation in increasing the competitive capacity of companies that participate in subversion programs and that are able to develop, such as those that participate in technological development clusters. with its effective capacity gain after the end of its participation in the program.In this respect, Brazil acts more strongly with programs that aim to develop the competitive capacity of companies: National Program for Exporting Culture, Industrial Extension Program for Export, More Brazil, Local Innovation Agents, among others. These aim to develop the competitive capabilities of the selected companies in order to operate at a high level of competitiveness. Thus, this research aims to analyze the competitive capacity focused on the internationalization of micro and small companies that participated in incentive programs to increase competitiveness and to identify which capacities were included in their market performance strategy at the end of the program that indicates the effectiveness of the program. For this, 256 companies participating in these programs are analyzed based on the data obtained in the reports of these programs.

Keywords: small and medium-sized companies; internationalization; competitiveness; innovation.

\footnotetext{
${ }^{1}$ Mestra em Propriedade Intelectual e Transferência de tecnologia / Universidade Federal de Pernambuco - UFPE / Recife, Pernambuco Brasil

${ }^{2}$ Doutor em Engenharia Elétrica / Universidade Federal de Pernambuco - UFPE / Recife, Pernambuco - Brasil

${ }^{3}$ Mestra em Ciência da Computação / Universidade Federal de Pernambuco - UFPE / Recife, PPernambuco - Brasil
} 
1 Introdução

As Micro e Pequenas Empresas (MPE) vêm progressivamente aumentando sua relevância na economia brasileira. Verificou-se, através da sua evolução na participação no Produto Interno Bruto (PIB), que as MPE passaram de 21\% em 1985 29,5\% em 2019, levando-se em conta que as mesmas representam 99\% das empresas existentes no país. Esta participação encontra-se em constante adequação aos movimentos da economia, observando que há uma tendência de crescimento nos setores de atividades econômicas, tanto em serviços como no comércio, com menor participação no setor industrial, onde predominam médias e grandes empresas que se beneficiam de economia de escala (Serviço Brasileiro de Apoio às Micro e Pequenas Empresas [SEBRAE], 2020).

Nos países economicamente desenvolvidos, as MPE representam 98\% das empresas existentes no mercado; são responsáveis por oferecerem $60 \%$ dos empregos e contribuem com $50 \%$ do PIB. No entanto, nos países de economias em desenvolvimento, essas empresas de menor porte conseguem participação da ordem de 30\% nos empregos e sua representatividade no PIB revela-se na ordem de 10\% (Organização para Cooperação e Desenvolvimento Econômico [OCDE], 2020).

Entretanto, para afastarem as ameaças impostas pelo mercado e se manterem competitivas, as MPE precisam, além de inovar, obterem ganhos da capacidade competitiva. Cada organização deve ser capaz de reconhecer os seus pontos fortes e fracos para estabelecer estratégias inovadoras, ter flexibilidade e agilidade para atender a novos requisitos do mercado no tempo certo, qualidade dos produtos e serviços a partir da percepção dos clientes, além de atuar com uma estratégia de marketing que permita a potências compradores perceberem os diferenciais competitivos associados ao produto e a sua marca (OCDE, 2020). Assim, o ganho de capacidade competitiva passa a ser elemento fundamental para as organizações que buscam as ampliações de suas atuações nos mercados e obtenção de diferenciais competitivos, ao mesmo tempo em que se observa a necessidade de desenvolver modelos de educação que estejam alinhados com as necessidades de formação profissional. Esta ação precisa ser estimulada com ofertas de formações prioritariamente em tecnologias para permitirem ampliações nos setores de atividades econômicas com capacidades competitivas diferenciadas e de alto valor agregado.

Nesse contexto, observa-se que o Brasil é um país atraente para o mercado internacional nos diversos setores de atividades econômicas. Uma vez que, apresenta alta vulnerabilidade frente ao mercado globalizado, pois, são poucas indústrias nacionais de alta tecnologia com capacidade exportadora e poucas marcas nacionais com reconhecimento internacional. Conduzindo assim a necessidade de se estabelecer estratégias que permitam a criação de um ecossistema favorável à inovação tecnológica, que facilitaria o surgimento de incubadoras vinculadas às instituições de ensino superior apoiadas por políticas públicas capazes de permitirem o surgimento de centenas de milhares 
de startups. Assim, surge a necessidade do suporte através de incentivos capazes de propulsar a criação de empregos e renda. E ainda como uma solução da parte do problema que trata da vulnerabilidade do mercado de produtos industrializados no Brasil, devido a sua pouca presença no mercado exterior.

Como percebido que a maioria das empresas em todo o mundo é Micro e Pequenas Empresas (MPE) e assim sendo, apresentam limitações e necessitam de um ambiente que Ihes permitam desenvolvimento das suas capacidades competitivas. Assim, é observado que em toda nação desenvolvida ou em desenvolvimento existe um ecossistema que seja favorável aos empreendimentos que surgem e que lhes permitiram ampliar as suas participações na economia, de uma região ou nação. Para que isso aconteça se faz necessário criar a infraestrutura, capacidades intelectuais (talentos), formação de pessoas e estimular com financiamentos e subvenção. Essas proposições estão postas na literatura e em estudos atuais sobre empreendedorismo, inovação e startup (Kao, 2008). Porém, devese considerar a existência de várias iniciativas no Brasil que se adota com a proposição de acelerar esse processo considerando que já existem muitas incubadoras espalhadas no país. Neste contexto, considerando a existência de um grande número de micro e pequenas empresas (MPE) no Brasil foram desenvolvidas iniciativas, tais como Projeto para Desenvolvimento Nacional da Cultura Exportadora (PNCE) e o Programa de Extensão Industrial para Exportação (PEIEX), que buscam atuar junto às empresas com potencial de exportação, subsidiar consultorias, treinamentos e financiamentos para desenvolverem ganhos de capacidades competitivas com vistas ao desenvolvimento da cultura de exportação através de ações de negócios de exportação. Esperando-se que após a primeira exportação essas empresas desenvolvam trilhas exportadoras, ampliando os seus negócios com produtos, serviços e logísticas adequadas. Identificam-se a falta de estudos científicos que façam avaliações que indique a melhor forma de promover essas empresas para um desempenho diferenciado desejado e exigido no mercado exterior.

Observa-se que as MPE têm no perfil de escolaridade de seu gestor 50\% com nível superior (SEBRAE, 2020). Faz-se necessário observar qual é perfil das empresas que participam desses programas e os impactos causados considerando os aspectos associados a indicação de que essas empresas irão continuar exportando ou buscado exportar após a sua finalização no programa. Assim, busca-se evidenciar a necessidade estrita por um ambiente de desenvolvimento permanente até tornarem-se empresas de porte médio. Para efeito dessa pesquisa, tomou-se por base o projeto PEIEX que ocorreu no estado de Pernambuco no período de 2016-2018 com a participação de 256 empresas localizadas na região metropolitana da cidade de Recife. Como resultado obtém-se os tipos de ações realizadas em cada empresa observando-se o nível de capacidade competitiva percebida e a partir dessa percepção estabelecer o nível de maturidade médio. Dando indicativo da dificuldade dessas empresas serem bem sucedidas quando da adoção da cultura da exportação desde que não sejam mantidas em um ambiente de propulsão de inovação (Kao, 2008). 


\section{Referencial Teórico}

Neste capítulo são apresentadas definições de vantagem competitiva e internacionalização de empresas e de MPE. A categorização se mostra necessária por conta das peculiaridades encontradas na literatura a respeito desse modelo de empresas que apresenta características distintas que não as permite funcionarem como versões reduzidas de grandes empresas (Tunes \& Monteiro, 2017). Ainda são trazidos os conceitos de born global e borderless firms, por compreenderem novas organizações e modelos de internacionalização provenientes de ambientes dinâmicos.

\subsection{Vantagem competitiva}

Vantagem competitiva é um tema que vem sendo abordado por diversas correntes econômicas a partir dos anos 70 e, como Vasconcelos (2000) observa, algumas dessas correntes sugerem que a vantagem competitiva pode ser explicada por fatores externos como a dinâmica de mercado e a estrutura das indústrias. Por outro lado, existem correntes que procuram explicar a vantagem competitiva a partir de um conjunto de fatores internos específicos à firma.

De acordo com Mozzato \& Gzybovski (2018) a vantagem competitiva se configura como a capacidade de uma empresa gerar um valor diferenciado em seus produtos ou serviços frente aos demais concorrentes. Porter (2004) sinaliza que a vantagem competitiva poderia ser obtida através da adoção de estratégias como diferenciação, liderança de custos e enfoque que devem ser eleitas de acordo com o objetivo da empresa. O autor também defende que o posicionamento da empresa dentro do mercado é o principal determinante de sucesso no cenário competitivo.

Etemod (2017) defende que a partir da entrada em um mercado internacional, a empresa enfrentará novos desafios, pois mesmo que seus produtos e serviços se mantenham, a inserção em um novo ambiente geográfico trará novas variáveis que precisam ser consideradas no desenvolvimento da estratégia da empresa. Nesse caso ela precisará crescer a partir do enfrentamento dessa nova realidade. Os autores relacionam a diferenciação dos produtos e serviços com os pressupostos de Tunes \& Monteiro (2017), e no que diz respeito a diferenciação de mercado propondo a criação de um novo conceito de produtos e serviços para os clientes que estarão associados à sua marca.

A partir do desenvolvimento de uma vantagem competitiva, uma empresa não pode garantir que essa situação venha a se perpetuar. Por essa razão, vários autores discutem a forma mais eficiente de garantir a posição de destaque frente os concorrentes. Mozzato \& Gzybovski (2018) com a Resource Based View corroboram que a forma como os recursos empresariais são geridos explicam o seu desempenho para manterem os seus ganhos de vantagem competitiva.

A partir de então foi reformulado o modelo desenvolvido por eles. Este novo modelo estabelece que os recursos que uma empresa possui podem ser classificados a partir de quatro indicadores, 
tornando mais dinâmica a compreensão da real vantagem competitiva possuída. O modelo VRIO considera que esses recursos podem ser divididos em valiosos, raros, imitáveis e exploráveis pela organização e à medida que o recurso atinge patamares superiores, a vantagem competitiva pode ser sustentada pela organização (De Melo \& Leone, 2015).

A partir de Skoludova e Kozena (2015), pode-se observar a consonância com o pensamento De Melo e Leone (2015) de que a criação de vantagens competitivas é peça central para o sucesso de uma empresa em um cenário economicamente dinâmico. No entanto, a primeira análise de parte do pressuposto de que não apenas o lucro financeiro caracterizaria o sucesso dos negócios, mas também uma série de fatores que envolvem inovação, construção e promoção de marcas, flexibilidade e qualidade da produção coincidem com a criação de competitividade.

O estabelecimento de uma vantagem competitiva também pode ser relacionado à capacidade que uma empresa possui em adotar estratégias de inovação, muito embora envolva incertezas provenientes de sua complexidade (Tidd \& Bessant, 2015). De acordo com os autores, assim como a empresa precisa alinhar suas estratégias a um cenário de globalização, a fim de criar vantagem competitiva, o uso da inovação para detectar oportunidades e estabelecer relações é de extrema necessidade.

\subsection{Internacionalização de empresas}

A tendência da globalização de mercados, a partir dos anos 90, levou o Brasil a adotar outra abordagem em relação ao comércio exterior: "buscou-se elevar o nível de competição e qualidade das empresas brasileiras através de uma maior exposição internacional” (Kraus, 2006). Para Francischini, et al. (2015) esta era uma saída inevitável, pois as empresas que não adotaram a adaptação às realidades globais se tornaram vítimas das que aderiram a essa tática.

De acordo com Gohr \& Silva (2015) a discussão sobre internacionalização, gira em torno da ordenação dos "diferentes pedaços do mosaico" a fim de uma integração dos conhecimentos sobre os negócios internacionais. Destacam-se os trabalhos de Nascimento et al (2017) e Da Rosa et al. (2018) que além de ressaltarem a discussão sobre o tema propõem uma metodologia para mensurar o grau de internacionalização das empresas que se configuram como modelos de internacionalização.

De acordo com os dois modelos, os seis estágios de internacionalização vão desde a falta de interesse de exportação, por parte da empresa, até a construção de conhecimentos e posterior aplicação que proporcione a ela ser uma experiente exportadora (Da Rosa et al, 2018) que analisa a viabilidade de exportar para países psicologicamente mais distantes (Bikey \& Tesar, 1977). Essa teoria remonta ao modelo de internacionalização da Universidade de Uppsala.

O então modelo desenvolvido pela Universidade de Uppsala é composto de quatro estágios distintos que consistem em: 1) atividades não regulares de exportação; 2) exportação via 
representantes independentes; 3) implantação de uma subsidiária de vendas no exterior; e 4) produção no exterior ou implantação de unidade de manufatura. As atividades nos determinados estágios avançariam, como esquematizado por Heinzmann (2014), à medida que adquirisse maior conhecimento de mercado e incrementasse o comprometimento de recursos.

Outro viés que remete às contribuições do modelo Uppsala é o indicativo de atuação inicial em países culturalmente e psiquicamente mais próximos. Isto diz respeito às características como o idioma de destino, educação, sistemas políticos e econômicos, cultura, práticas comerciais, nível de desenvolvimento industrial, entre outros aspectos que quanto mais semelhantes entre o país de origem e o de destino seriam indicativos de sucesso nas exportações (De Oliveira et al, 2018).

Os mesmos autores também remontam que entre os fatores motivadores da internacionalização está a abertura para novos mercados, aspecto intensificado quando ocorre o esgotamento das possibilidades internas. De acordo com a teoria do poder de mercado formulada por Domingues et al (2016), as empresas se dispõem ao risco da internacionalização visando acréscimo nos lucros após o esgotamento das oportunidades no mercado interno ou flutuações de marcado devido políticas econômicas locais.

Para Mcdowell et al (2016) quando uma empresa busca o caminho da internacionalização, ela participa de um processo composto por cinco fases que inicialmente parte do pressuposto da decisão de ingressar no mercado internacional. A partir da escolha pela internacionalização, outras decisões precisam ser tomadas como o mercado que decidirá se inserir e a modalidade que irá ingressar, bem como alinhar estratégias sobre o programa e organização de marketing.

O autor também estabelece que o processo de internacionalização pode acontecer em quatro etapas, iniciando com as atividades de exportação inconstantes, posteriormente a exportação via representantes independentes, o estabelecimento de subsidiárias de vendas e estabelecimento de instalações de produção no exterior. Essas fases remetem ao modelo Uppsala e, nesse sentido, Kotler (2000) também defende que as decisões de internacionalização devem ser tomadas de forma progressiva.

Apesar da proposição e discussão sobre os diferentes modelos de internacionalização, de acordo com Toyne e Nigh (1997) ainda haveria dificuldade de generalização dos resultados de estudos, gerando uma lacuna nesse contexto. Os motivadores seriam a dificuldade resultante de envolver diferentes realidades históricas econômicas buscando avaliar uma realidade empresarial com pressupostos e conhecimentos de outra realidade (Kraus, 2000). 


\subsubsection{Born global e borderless firms}

A evolução do processo de internacionalização de empresas mostra que uma nova gama de empreendimentos apresenta estratégias de rápida expansão internacional desde a sua formação ou logo após a sua criação (Corado-Simões, Rocha, Mello \& Carneiro, 2015) em que suas trajetórias não seguem o tradicional modelo gradual de Uppsala (Johanson \& Vahlne, 1977; VahIne \& Johanson, 2017).

Por sua vez, as pesquisas sobre Empreendedorismo Internacional (El) surgiram no início dos anos 1990, buscando entender a natureza dinâmica de pequenas empresas precocemente internacionalizadas, utilizando-se de perspectivas teóricas do campo de negócios internacionais, empreendedorismo, gerenciamento estratégico, redes de relacionamentos e marketing (Peiris, Akoorie \& Sinha 2012).

Embora tenham sido nomeadas de diferentes formas, essas empresas ficaram comumente conhecidas como International New Ventures (INV) através do trabalho de Oviatt e McDougall (1994) e também como Born Globals (BG) (Rennie, 1993; Knight \& Liesch, 2016), ainda que alguns autores considerem ser a BG apenas um tipo de INV (Coviello, 2015).

Para alguns autores a definição de Born Globals, Global Start-ups e International New Ventures referem-se a "organizações que, desde ou próximo a sua concepção, buscam alcançar um resultado internacional superior através da aplicação de recursos baseados em conhecimento para comercializar seus produtos em múltiplos países" (Knight \& Cavusgil, 2004, p. 124).

De acordo com Oviatt e McDougall (1994, p. 49), essas terminologias configuram "organizações que, desde a sua origem, almejam adquirir vantagens competitivas da utilização de seus recursos e da venda de sua produção em diversos países". Ressaltando que não é necessário que haja algum tipo de investimento externo direto, podendo essas vantagens se tratar apenas da formação de alianças internacionais para produção, marketing ou vendas.

Simões et al. (2015) argumentam que existem INV que desafiam a lógica das empresas de origem bem definida, empresas que são desenvolvidas desde o início com operações, recursos e capacidades dispersos geograficamente, que possuem diversos motivos além da busca por novos mercados para se internacionalizar, e que deveriam, portanto, ser chamadas de Borderless Firms (BF), ou "empresas sem fronteiras".

Essas empresas poderiam ser identificadas pela combinação entre as seguintes características (Simões et al., 2015, p. 182): 1) atividades da cadeia de valor espalhadas em diversos países e regiões desde a sua concepção (ou pouco tempo depois da sua criação); 2) empreendedores não restritos cognitivamente a uma base de operações; fundadores ou times de gerências multinacionais; 3 ) força de trabalho multinacional. 
Para Etemad (2017), as BF são empresas que se desenvolveram de forma que sua cadeia logística está distribuída em regiões que oferecem as melhores vantagens comparativas para cada atividade. Essa configuração proporciona um aumento na competitividade geral da empresa e na sua capacidade de entregar produtos de maior valor agregado a seus clientes, tornando mais fácil a comercialização no mercado global.

\subsection{Internacionalização de MPE}

A internacionalização das micro e pequenas empresas (MPE) por meio das exportações é uma estratégia relevante para que este segmento inove e obtenha vantagem competitiva. A entrada das MPE no mercado externo via exportação é um modo que oferece um maior grau de flexibilidade, visto que o sucesso de exportação para as MPE é relevante para os governos e contribui para o desenvolvimento econômico das nações (Sousa \& Novello, 2014).

Devido a sua relevância, as pesquisas de fatores de sucesso e o desempenho exportador de MPE têm recebido atenção em áreas que buscam compreender seu papel nas economias e oportunidades no mercado externo (Haahti et al., 2005). As MPE não operam como versões menores das grandes companhias por possuírem características diferentes como o ambiente no qual estão inseridas, o estilo gerencial, a estrutura proprietária, a independência, a escala e o escopo da operação (Brouthers \& Nakos, 2004; Zhao, M. et al. 2018).

Segundo o SEBRAE (2020), o aumento do número de MPE exportadoras em 2019 repetiu o crescimento observado em 2014, 2015 e 2016. A evolução histórica do número de empresas exportadoras entre 2009 e 2019, evidenciando que, neste último ano, registrou-se número recorde de MPE exportadoras da série, superando, pela primeira vez, o patamar de 8 mil empresas. Considerando apenas o último quadriênio, o aumento acumulado do número de microempresas exportadoras foi de 19,3\%, e o de pequenas empresas foi ainda mais expressivo, de $39,8 \%$, em ambos os casos revertendo uma tendência de ligeira queda entre 2015 e 2019. Entre as empresas médias e grandes, o crescimento no quadriênio foi mais modesto $(7,9 \%)$, mas também se atingiu número recorde de empresas exportadoras em 2019.

Comparando as estratégias utilizadas pelas multinacionais e pelas MPE na busca da internacionalização, Ruzzier, Antončič e Konečnik (2006) definem as diferenças adotadas por essas empresas. As multinacionais explorariam questões como estratégias globais, alianças internacionais e problemas de diversificação e controle; as MPE focariam em um processo incremental de internacionalização. Por essa razão, o modelo Uppsala foi adotado como base teórica para o desenvolvimento da maior parte das pesquisas sobre sua trajetória de internacionalização.

Boehe et al (2011) relacionam o tamanho da empresa com a propensão para a exportação e em seus estudos, este tópico supera o efeito das relações entre organizações locais. Outro fator que 
impacta na menor participação de empresas menores nas exportações nacionais é a aversão ao risco que pode vir a ser proveniente da limitação de informações e menor disponibilidade de recursos financeiros e profissionais preparados para o mercado externo (Calof, 1994). Por outro lado, alguns autores defendem que embora os estudos empíricos realizados com as MPE mostrem uma adequação ao processo incremental de internacionalização, aspectos como o ritmo e padrões de crescimento internacional, escolha do mercado e do modo de entrada em outros países são altamente influenciados pelas relações estabelecidas por essas empresas, seus clientes e fornecedores (Bonaccorsi, 1992; Bell, 1995; Coviello \& Munro, 1997).

Outro ponto relevante no processo de internacionalização das MPE é a utilização de redes de relacionamento internacionais, ao invés de seguir o modelo incremental para entrar no mercado internacional. Os estudos de Coviello e Munro (1997), com base nas MPE de tecnologia, sugerem que o rápido acesso ao mercado internacional se deu a partir de uma variedade de mecanismos, além da participação em redes de relacionamento internacionais.

O avanço das pesquisas na área de internacionalização de MPE mostra que, majoritariamente, esses novos empreendimentos não acompanham o modelo Uppsala (Oviatt \& Mcdougall, 1994; Knight e Cavusgil, 2004), buscando diferentes estratégias para atingir o mercado internacional. Nesses estudos são destacados o papel do empreendedor na interação social, a formação de redes e o uso da visão global na formulação de estratégias e de cultura organizacional.

Nesta mesma linha de pensamento, Rennie (1993) publicou sua pesquisa sobre empresas australianas cujo objetivo principal era a exportação, e cunhou o termo Born Globals, enquanto o artigo seminal de Dzikowski (2018) fornece um enquadramento que busca explicar o comportamento dos International New Ventures, integrando conceitos das teorias de negócios internacionais com o desenvolvimento de pesquisas sobre o empreendedorismo.

Os autores enfatizam desde o princípio a importância do conhecimento e outras capacidades organizacionais únicas para explicar o rápido processo de internacionalização dessas empresas. Knight e Cavusgil (2004) também afirmam que falta de experiência e escassez de recursos financeiros, humanos e tangíveis não se configuram como barreiras à internacionalização em grande escala, fazendo-os serem vistos como um sistema de negócios diversificado no qual qualquer empresa pode ser bem-sucedida internacionalmente.

\section{Metodologia}

Esta pesquisa é de caráter exploratório, pois proporciona maior aproximação com o experimento permitindo maior compreensão do problema (Gil, 2002, p. 41). O estudo possui natureza quantitativa sendo elencada, ademais por não permitir que crenças e valores exerçam maior influência que a teoria, conforme Günther (2006). Sendo desenvolvida com base no cruzamento da teoria de 
Skoludova e Kozena (2015) sobre a mensuração da competitividade e as informações levantadas a partir dos diagnósticos e relatórios de atendimento das empresas que participaram do Programa de Qualificação para Exportação (PEIEX) no Núcleo Recife entre os anos 2016 e 2018.

A amostra não aleatória é utilizada por conta da conveniência de se tratar de um grupo de 256 empresas que participaram do programa no período selecionado (Coutinho, 2014). Conta-se majoritariamente com a participação MPE dos setores de alimentos e bebidas, têxteis, metalurgia, biotecnologia, tecnologias e sistemas de informações, entre outros que permite a investigação acerca da capacidade competitiva das MPE que buscam o caminho da exportação.

De acordo com a metodologia PEIEX é a partir da Matriz de Identificação Estratégica que se pode visualizar de forma gráfica o posicionamento estratégico da empresa, auxiliando a optar pelas melhorias para implantar com propósito de obter ganhos de capacidade competitiva. Informando sobre possíveis indicações de atuação nas áreas como Administração Estratégica, Capital Humano, Finanças e Custos, Vendas e Marketing, Comércio Exterior e Produtos e Manufaturas, cada uma delas com subitens onde são declarados aspectos sobre planejamento e indicadores de desempenho, entre outros classificados de acordo com seu status e importância sintetizados no Quadro1.

Nos subitens da dimensão Comércio Exterior, nomenclaturas próprias são definidas com a finalidade de criar uma maior dinâmica em relação as recomendações de planejamento de ações. Algumas delas são usadas exclusivamente pelo programa e outras foram ressignificadas a partir das proposições pré-definidas pela APEX- Brasil.

Entre os principais fatores de competitividade selecionados por Skoludova e Kozena (2015) estão inovação, construção e promoção de marcas, flexibilidade e qualidade da produção. Esses itens estão descritos no Quadro 2 que se relacionam com as melhorias PM 2, VM 2, AE 1 e PM 3, respectivamente, como mostrado nos itens da matriz de identificação estratégica do PEIEX no Quadro 1. 


\section{Quadro 1}

Dimensões da matriz de identificação estratégia e campos de melhorias do PEIEX

\begin{tabular}{|c|c|}
\hline Dimensão & Melhoria \\
\hline \multirow{3}{*}{ ADMINISTRAÇÃO ESTRATÉGICA (AE) } & Estratégia organizacional (AE 1) \\
\hline & Estrutura organizacional (AE 2) \\
\hline & Indicadores de desempenho (AE 3) \\
\hline \multirow{4}{*}{ CAPITAL HUMANO (CH) } & Estratégias de recursos humanos ( $\mathrm{CH} 1$ ) \\
\hline & Administração de recursos humanos (CH 2) \\
\hline & Educação e treinamento $(\mathrm{CH} 3)$ \\
\hline & Indicadores de recursos humanos ( $\mathrm{CH} 4)$ \\
\hline \multirow{4}{*}{ FINANÇAS E CUSTOS (FC) } & Administração financeira (FC 1) \\
\hline & Indicadores financeiros (FC 2) \\
\hline & Administração de custos (FC 3) \\
\hline & Indicadores de custos (FC 4) \\
\hline \multirow{3}{*}{ VENDAS E MARKETING (VM) } & Avaliação de mercado (VM 1) \\
\hline & Política mercadológica (VM 2) \\
\hline & Análise de desempenho (VM 3) \\
\hline \multirow{4}{*}{ COMÉRCIO EXTERIOR (CE) } & Engenharia de exportação (CE 1) \\
\hline & Plano de internacionalização da empresa (CE 2) \\
\hline & Consórcio de exportação (CE 3) \\
\hline & Processo de exportação (CE 4) \\
\hline \multirow{6}{*}{ PRODUTO E MANUFATURA (PM) } & Desenvolvimento de produto (PM 1) \\
\hline & Gestão da inovação (PM 2) \\
\hline & Processo e manufatura (PM 3) \\
\hline & Qualidade e indicadores (PM 4) \\
\hline & Gestão ambiental (PM 5) \\
\hline & Eficiência energética (PM 6) \\
\hline
\end{tabular}

Fonte: Elaborado pelos autores 
Quadro 2

Dimensões de análise da competitividade e suas definições

\begin{tabular}{|c|c|}
\hline Dimensão & Definição \\
\hline Inovação & $\begin{array}{l}\text { Permite que a empresa reaja com destreza às dinâmicas no } \\
\text { ambiente e economia }\end{array}$ \\
\hline $\begin{array}{l}\text { Construção e promoção de } \\
\text { marcas }\end{array}$ & $\begin{array}{l}\text { Refere-se ao acréscimo de um sinal de identificação no produto } \\
\text { que adiciona um novo valor. }\end{array}$ \\
\hline Flexibilidade & Nível de prontidão e agilidade para reagir às mudanças. \\
\hline Qualidade da produção & $\begin{array}{c}\text { Acréscimo de características nos produtos em cenários em que a } \\
\text { oferta excede a demanda. }\end{array}$ \\
\hline
\end{tabular}

Fonte: Adaptado de Skoludova e Kozena (2015).

Os requisitos do Plano de Internacionalização (CE 2) e do Processo de Exportação (CE 4) definidos na metodologia do PEIEX podem ser relacionados com os pressupostos de Kotler (2000). No que diz respeito à decisão de internacionalização, o autor sugere o processo composto pelas cinco fases de decisão a respeito do ingresso no mercado internacional que remontam o plano indicado em CE 2, onde se discute alternativas de ingresso, em qual mercado irá ingressar entre outras estratégias.

O autor também estabelece que o processo de internacionalização pode acontecer em quatro etapas distintas, desde a atividade de exportação inconstante até o estabelecimento de instalação de produção externa. Esses pontos que ilustra o que é visto em CE 4 e também são citados por Johanson e Wiedersheim-Paul (1993), juntamente com a gradualidade de comprometimento das atividades e remontam os pressupostos do modelo de internacionalização de Uppsala.

\section{Resultados}

O total de empresas atendidas pelo PEIEX em Recife foi um total de 256 empresas. Durante o atendimento houve recomendação de um total de 4.465 ações a fim de orientar essas empresas rumo à exportação. Dessas, aproximadamente 99\% foram atendidas, resultando em 4.220 demandas efetivadas pelo programa.

As recomendações de atendimento para Produtos e Manufatura estabeleceram-se como a mais numerosa em indicações de melhorias, contabilizando 33\%. As recomendações de Comércio Exterior somaram 30\% das demandas. Administração Estratégica, Vendas e Marketing, Finanças e Custos e Capital Humano totalizaram, respectivamente, 19\%, 12\%, 5\% e 2\% das demandas, como mostrado na Figura 1. 
Figura 1

Demandas de acordo com as dimensões do diagnóstico

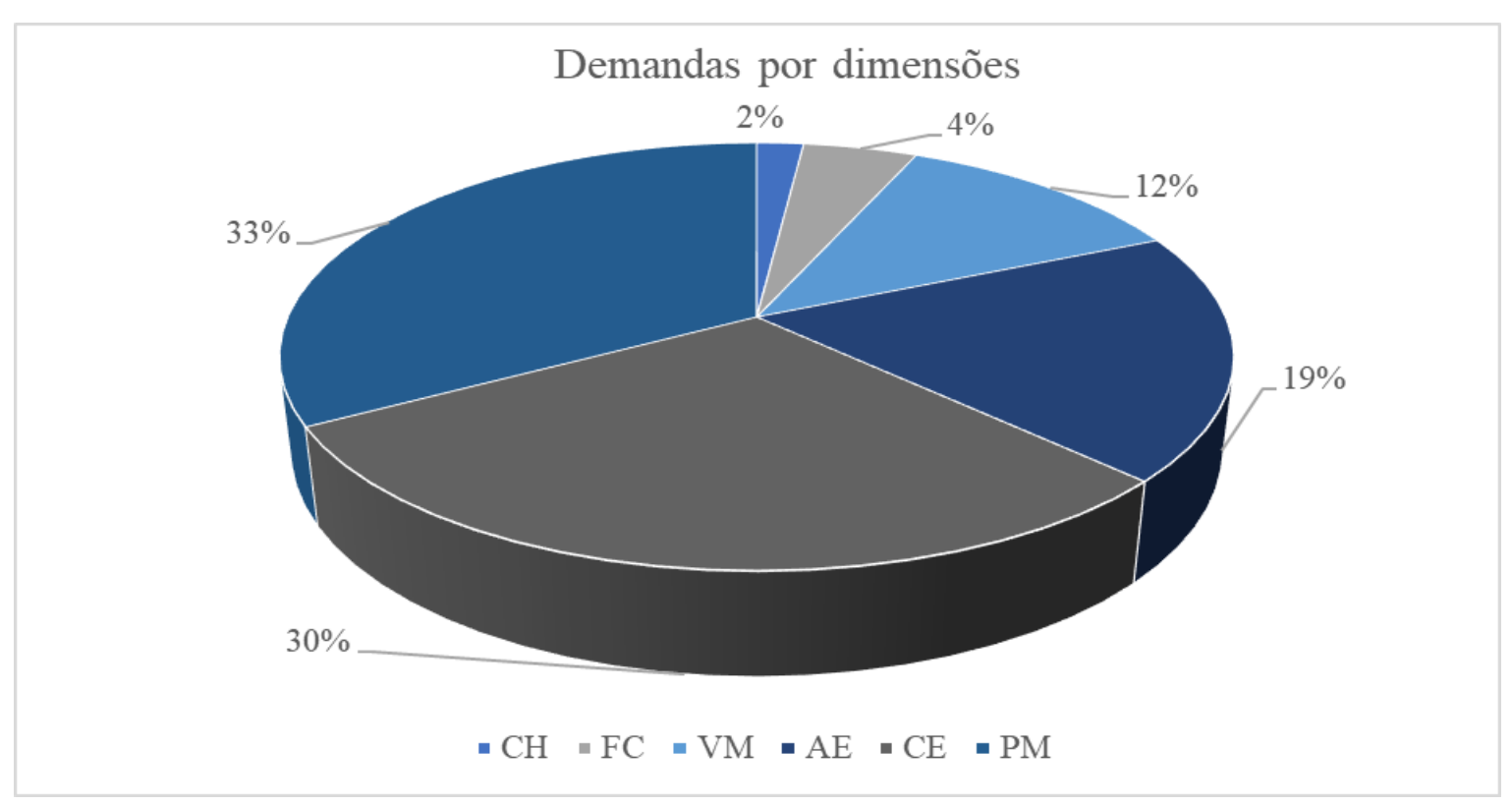

Fonte: MCTI (03/2016 - 06/2018).

Com relação às demandas atendidas, 64\% foram efetuadas nos próprios Núcleos Operacionais, por meio do técnico extensionista que estava atendendo a empresa ou demais integrantes da equipe técnica. Os 36\% remanescentes foram implantados por entidades parceiras como bancos e agências de desenvolvimento, federações da indústria, entre outros.

A partir do Quadro 3 pode ser visualizado o quantitativo de indicação de demandas entre os itens das suas áreas abrangidas no PEIEX. 


\section{Quadro 3}

Quantidade de melhorias indicadas por item

\begin{tabular}{|c|c|}
\hline Melhorias indicadas pelo PEIEX & Quantidade de Indicações \\
\hline Estratégia organizacional (AE 1) & 425 \\
\hline Estrutura organizacional (AE 2) & 30 \\
\hline Indicadores de desempenho (AE 3) & 335 \\
\hline Estratégias de recursos humanos ( $\mathrm{CH} 1$ ) & 15 \\
\hline Administração de recursos humanos $(\mathrm{CH} 2)$ & 25 \\
\hline Educação e treinamento $(\mathrm{CH} 3)$ & 20 \\
\hline Indicadores de recursos humanos $(\mathrm{CH} 4)$ & 20 \\
\hline Administração financeira (FC 1) & 65 \\
\hline Indicadores financeiros (FC 2) & 60 \\
\hline Administração de custos (FC 3 ) & 25 \\
\hline Indicadores de custos (FC 4) & 45 \\
\hline Avaliação de mercado (VM 1) & 360 \\
\hline Política mercadológica (VM 2) & 120 \\
\hline Análise de desempenho (VM 3) & 25 \\
\hline Engenharia de exportação (CE 1) & 105 \\
\hline Plano de internacionalização da empresa (CE 2) & 775 \\
\hline Consórcio de exportação (CE 3) & 350 \\
\hline Processo de exportação (CE 4) & 25 \\
\hline Desenvolvimento de produto (PM 1) & 540 \\
\hline Gestão da inovação (PM 2) & 325 \\
\hline Processo e manufatura (PM 3 ) & 365 \\
\hline Qualidade e indicadores (PM 4) & 145 \\
\hline Gestão ambiental (PM 5) & 15 \\
\hline Eficiência energética (PM 6) & 5 \\
\hline
\end{tabular}

Fonte: MCTI (03/2016-06/2018).

Na Figura 2 podemos verificar o resultado da demanda da área funcional Produto e Manufatura (PM), área que contabiliza o maior número de recomendações de demandas. As ações alocadas nessa área que receberam maiores indicações de melhoria foram desenvolvimento dos produtos (PM 1) que recebeu 39\% dessas indicações, seguido por processo e manufatura (PM 3), com 26\% das indicações; gestão da inovação (PM 2) com 23\% das recomendações. Os itens como qualidade de indicadores (PM 
4), gestão ambiental (PM 5) receberam por sua vez $11 \%$ e $1 \%$ respectivamente. 0 item eficiência energética (PM 6) não chegou a ter porcentagem expressiva mediante os demais valores.

\section{Figura 2}

Análise da área funcional Produto e Manufatura (PM)

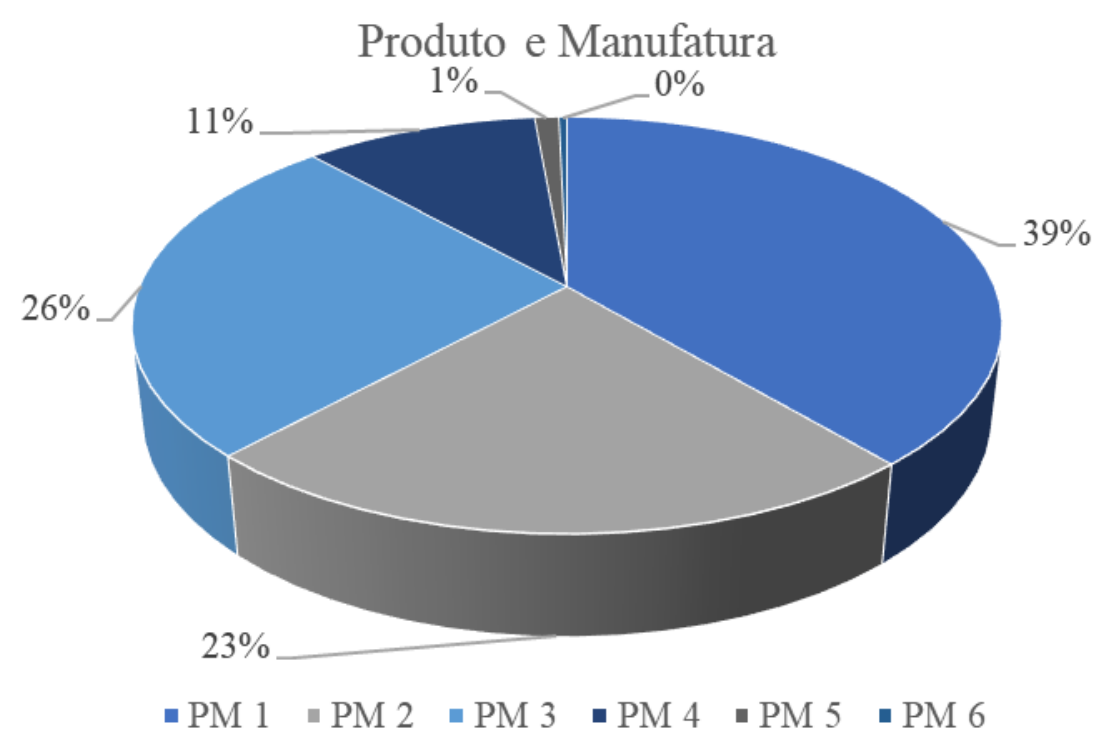

Fonte: MCTI (03/2016 - 06/2018).

A partir da análise da área de Comércio Exterior na Figura 3 verificou-se que a demanda mais numerosa se configura na elaboração de um plano de internacionalização da empresa (CE 2), mostrando a recomendação a $62 \%$ das empresas pesquisadas, assim como a necessidade de um consórcio de exportação (CE 3) às empresas que foram atendidas que representou $28 \%$ das indicações de atuação. Entre os itens com menores recomendações de melhorias estão engenharia de exportação (CE 1) com $8 \%$ e processo de exportação (CE 4) com $2 \%$ das recomendações de melhorias. 
Figura 3

Análise da área funcional Comércio Exterior (CE)

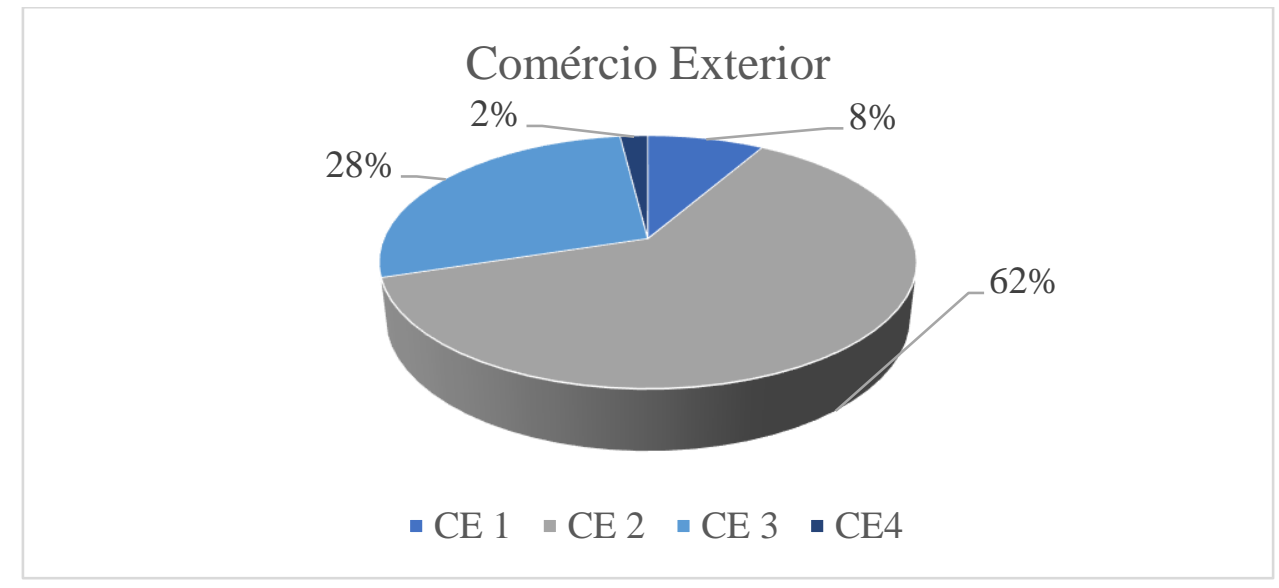

Fonte: MCTI (03/2016-06/2018).

Constata-se na Figura 4, que das demandas totais de Administração Estratégica, 54\% delas dizem respeito a melhorar ou implantar ações de estratégia organizacional (AE 1). Com relação a estrutura organizacional (AE 2), observa-se que $42 \%$ das empresas pesquisadas precisam de melhoria nesta área e 4\% demandaram ações para implantação de indicadores de desempenho (AE 3).

Figura 4

Análise da área funcional Administração Estratégica (AE)

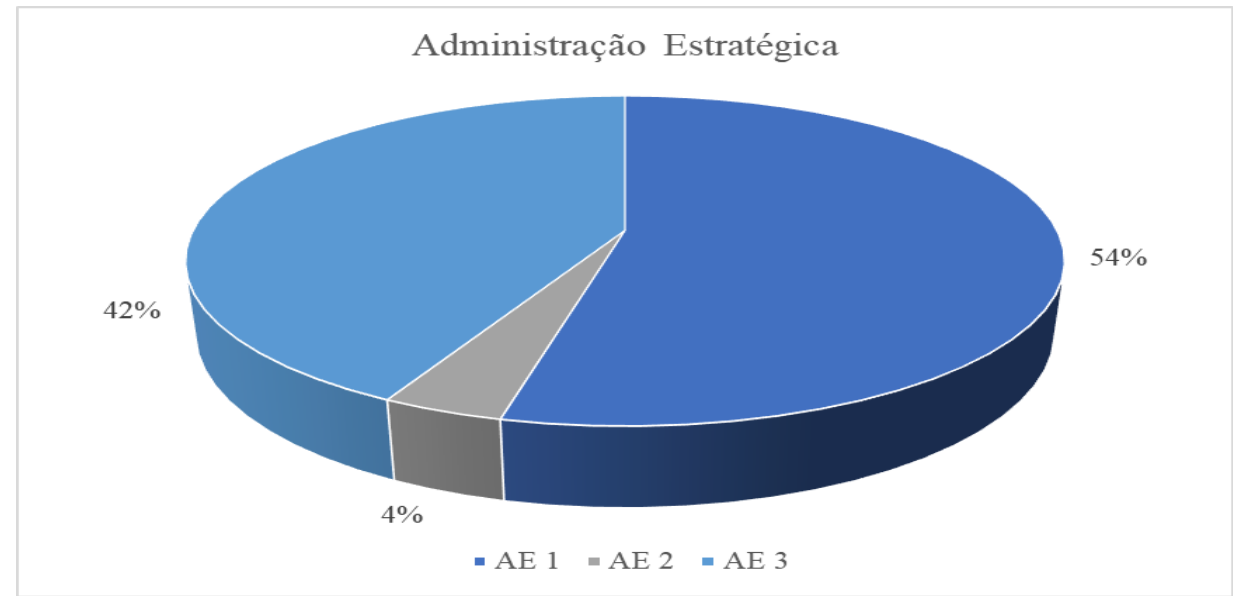

Fonte: MCTI (03/2016-06/2018). 
A partir da análise da dimensão Vendas e Marketing na Figura 5, pode-se ver que entre os destaques de melhoria estão a avaliação de mercado (VM 1) com $71 \%$ das recomendações seguido por política mercadológica (VM 2) com $42 \%$ das recomendações e análise de desempenho com $5 \%$.

\section{Figura 5}

Análise da área funcional Vendas e Marketing (VM)

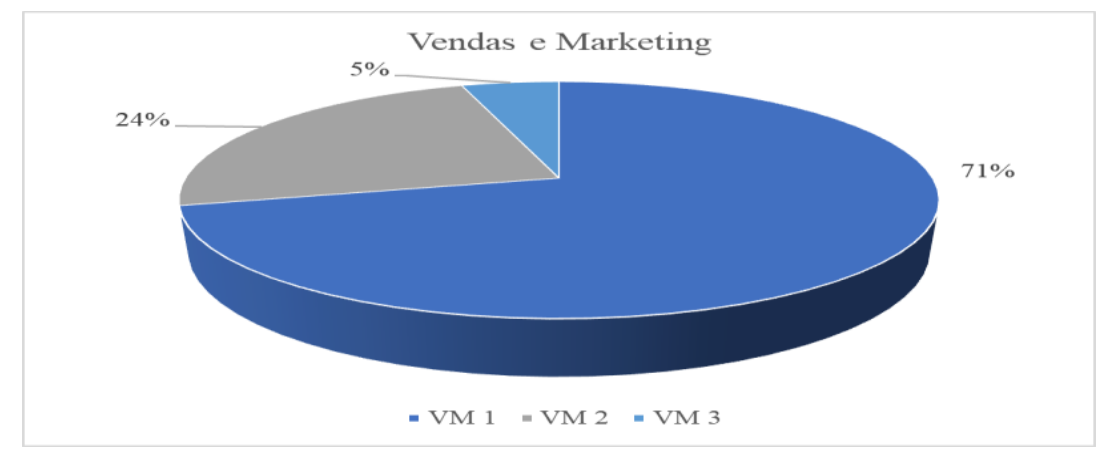

Fonte: MCTI (03/2016 - 06/2018).

$\mathrm{Na}$ análise dos dados correspondentes à área funcional Finanças e Custos, como pode ser visto na Figura 6, verificamos a maior incidência de indicações de melhorias para os itens administração financeira (FC 1) que obteve 33\% das indicações e indicadores financeiros (FC 2) com 31\% das indicações para esta área. Os itens administração de custos (FC 4) e indicadores de custos (FC 3) por sua vez receberam $23 \%$ e $13 \%$ das indicações, respectivamente.

\section{Figura 6}

Análise da área funcional Finanças e Custos (FC)

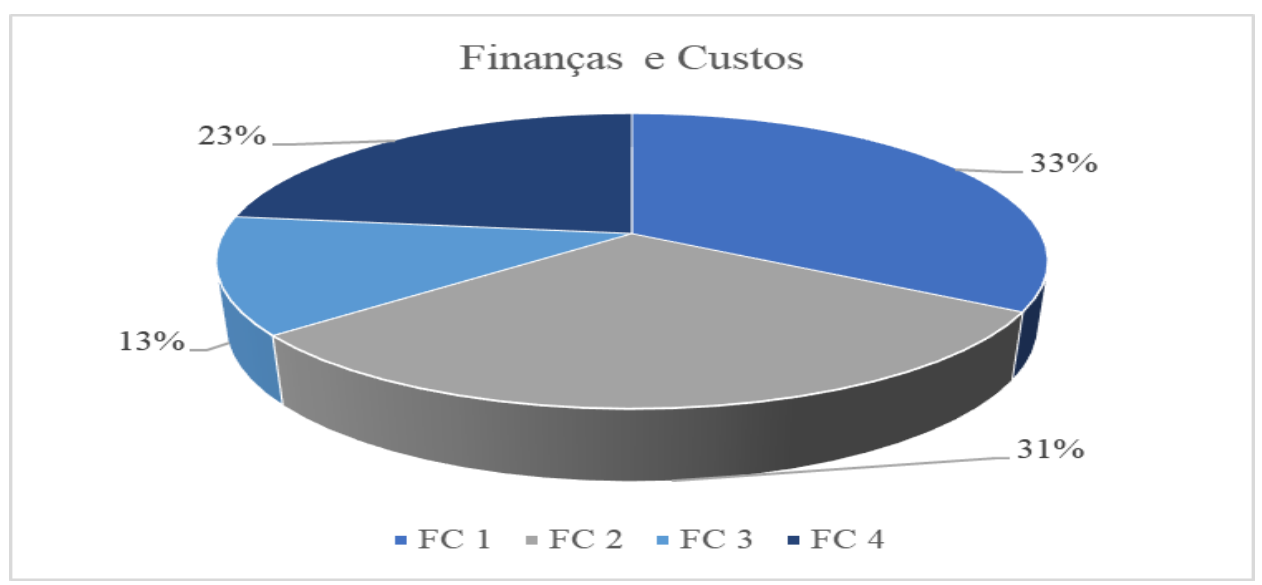

Fonte: MCTI (03/2016 - 06/2018). 
A Figura 7 corresponde à análise da área Capital Humano que recebeu o menor número de indicações de demandas em relação às demais $-2 \%$ do total de indicações de melhorias. Os itens apresentam-se da seguinte forma: administração de recursos humanos $(\mathrm{CH} 2)$ com $31 \%$ das indicações de melhorias; educação e treinamento $(\mathrm{CH} 3)$ e indicadores de recursos humanos $(\mathrm{CH} 4)$ contabilizaram 25\% das indicações de melhorias, cada; e estratégias de recursos humanos ( $\mathrm{CH}$ 1) com 19\% das indicações.

\section{Figura 7}

Análise da área funcional Capital Humano (CH)

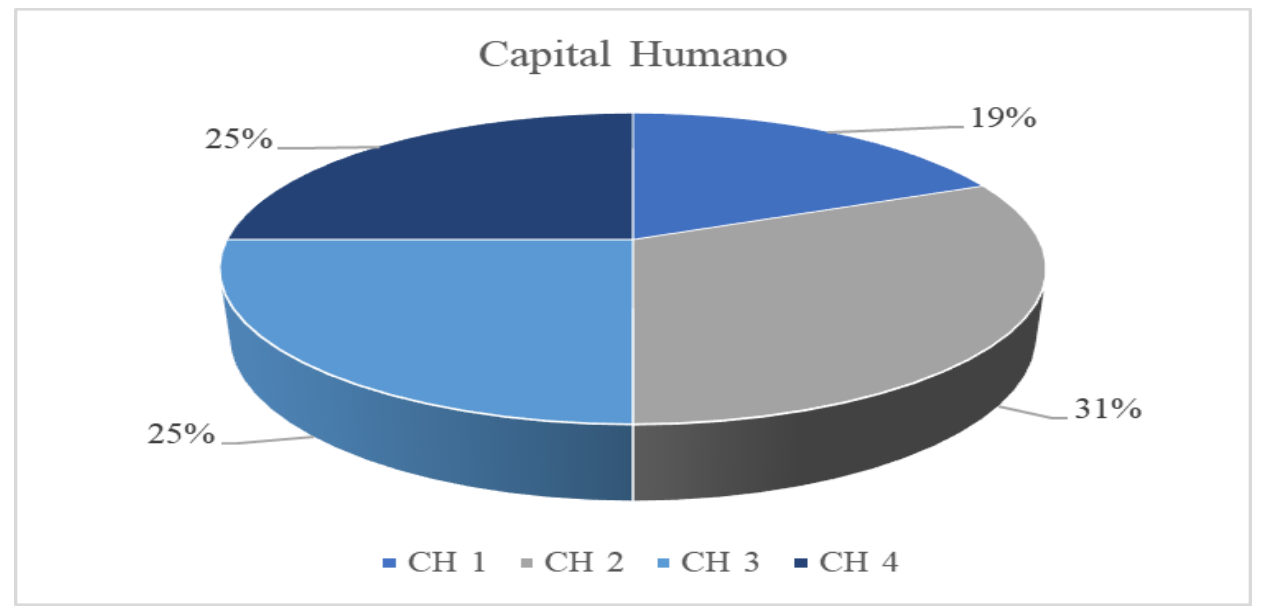

Fonte: MCTI (03/2016-06/2018).

\section{Discussão dos Resultados}

Inicialmente a partir da análise dos dados provenientes do PEIEX, um programa voltado para a qualificação de empresas para a exportação, inegavelmente o impacto das decisões sobre a área de Comércio Exterior se mostram como de extrema relevância, no entanto o fato de as demandas mais numerosas serem direcionadas à área Produto e Manufatura demonstra a necessidade de estabelecimento de estratégias acerca do produto anteriores ao processo de exportação em si, como posicionamento e manutenção no mercado interno, de acordo com a dimensão qualidade na produção elucidados por Skoludova e Kozena (2015).

Os itens presentes na área Comércio Exterior evidenciam a necessidade do desenvolvimento de um plano de internacionalização a partir do quantitativo de indicações de melhorias recomendadas para esse tópico, remontando o sugerido por Kotler (2000) no que diz respeito às decisões de internacionalização estarem alinhadas às demais decisões dentro da empresa, não podendo ser tomada de forma isolada. 
Com a análise da área Comércio Exterior também é possível perceber que a numerosa recomendação para melhoria de itens relacionados a Produto e Manufatura demonstra certa imaturidade na preparação das empresas participantes em realizarem de fato o processo de exportação, uma vez que a indicação para o processo de exportação (CE 4) contou com apenas $2 \%$ das indicações dentro desta área funcional e 25 indicações no quantitativo referente às demais demandas relacionadas às outras áreas.

A área Administração Estratégica nos mostra que entre os principais itens a serem indicados como merecedores de atenção para melhorias foram estratégia organizacional (AE 1) e estrutura organizacional (AE 2), juntos esses itens contabilizam 96\% das indicações de melhorias dentro da área Administração Estratégica. Esse quantitativo esclarece a extrema necessidade de adaptação das estruturas organizacionais de forma a promover uma estratégia mais eficiente em relação ao posicionamento no mercado internacional como podemos ver a partir de Kotler (2000) e Tidd \& Bessant (2015).

Finanças e Custos e Capital Humano foram as áreas que receberam o menor quantitativo de indicações de demandas. No entanto, quando analisamos os preceitos trazidos por Knight e Cavusgil (2004) verificamos que a escassez de recursos financeiros e humanos estão entre os itens que se configuram como barreiras à internacionalização em grande escala. A necessidade de maior atenção a esses itens mostrada na teoria e o pequeno quantitativo de indicações de melhorias nessas áreas pode ser reflexo do baixo desempenho de exportação dessas empresas participantes do PEIEX.

Os dados obtidos a partir da análise da área Vendas e Marketing trazem altos índices de indicação de melhorias para os itens avaliação de mercado (VM 1) e política mercadológica (VM 2). Esses itens apresentam relação com os indicadores propostos por Skoludova e Kozena (2015) no que diz respeito a construção e promoção de marcas no que tange a possibilidade acréscimo de características que indiquem aumento do valor do produto.

O fato de as demandas mais numerosas terem sido indicadas ao acompanhamento do próprio Núcleo Operacional - aproximadamente 64\% - representa a maior necessidade de interação entre as entidades envolvidas. Assim também podemos salientar que, do total de demandas sinalizadas pelos técnicos extensionistas e priorizadas junto com os empresários como de fato necessárias, 45 delas não foram efetivadas. Apesar de representar um percentual relativamente discreto em relação ao total de demandas que foram efetivadas - aproximadamente $1 \%$ - demonstra a necessidade de maior comprometimento das partes envolvidas em relação ao cumprimento dos acordos estabelecidos.

Outro fator relevante é o baixo quantitativo de indicações de melhorias referentes aos itens qualidade de indicadores e análise de desempenho presentes em cinco das categorias analisadas. A importância dos indicadores pode ser esclarecida a partir da relação destes itens com a dimensão Flexibilidade proposta por Skoludova \& Kozena (2015). O acompanhamento desse índice remete ao 
nível de prontidão que a empresa possui para reagir às mudanças que por sua vez indicam a chance delas se mostrarem mais competitiva em relação às demais.

A partir da análise dos dados do PEIEX notamos a relação do baixo desempenho em exportação das empresas integrantes do programa e as características preconizadas na teoria. Os baixos quantitativos de indicações de melhorias para essas empresas em certos itens elucidados na literatura como imprescindíveis a um desempenho superior refletem a necessidade de alinhamento da estrutura do programa em relação a promoção de efetivos ganhos para as empresas.

\section{Considerações Finais}

Devido à importância das MPE na economia, principalmente na geração de emprego, algumas políticas públicas são desenvolvidas com objetivo de tornar essas empresas mais competitivas. O favorecimento por meio de benefícios que esse tipo de empresas recebe visa estimular, entre outros, a formalização de empreendimentos no país.

Neste sentido, esta pesquisa analisou uma amostra de 256 empresas que participaram do Programa de Qualificação para Exportação (PEIEX) com objetivo de identificar a importância de projetos dessa natureza que dão indicação que é possível através da criação de um ambiente adequado verificado através de suporte técnico e financeiro ser possível desenvolver nas MPE ganhos de capacidades competitivas.

A partir dos resultados obtidos, verifica-se que investimentos em áreas como inovação, promoção de marcas, qualidade na produção são de extrema relevância na criação de vantagens competitivas para essas empresas. Concluí-se, ainda, que recomendações de investimento em comércio exterior não se configuram como ações isoladas para as empresas que decidem exportar. Observa-se que entre o planejamento de ações para exportação devem ser consideradas a capacidade competitividade associada a financeira para investir e atingir níveis de produtividade capazes de atenderem as demandas provenientes das provocações decorrentes das ações voltadas para exportação.

Daí resgata-se a questão que aparece como uma grande lacuna na literatura sobre quem é o grande financiador da inovação para conquista de novos mercados com exportação de produtos de alto valor agregado. Então, observa-se naqueles países que são líderes de exportação de produtos de alto valor agregado que a inovação é financiada ou subsidiada pelo poder público. Este financia a pesquisa e desenvolvimento através de institutos de pesquisa e universidades entregando ao setor privado a exploração de mercado. Para isso são criados ambientes controlados voltados para atenderem demandas de inovação que acolham as estratégias de competitividade de um país. Sendo assim, de interesse público trazendo no seu bojo competitividade no mercado privado gerando riqueza e empregos para o país. 
Com da análise dos dados de sugestões de melhoria do PEIEX foi possível perceber que a alteração no nome do programa - Projeto Extensão Industrial Exportadora para Programa de Qualificação para Exportação - ocorreu devido às demandas de muitas as empresas participantes precisarem de ajustes nas estruturas internas antes de iniciarem suas atividades no exterior. É possível perceber esta alegação pelo quantitativo de demandas para a área de Administração Estratégia (AE) ser superior a Comércio Exterior (CE), além das numerosas indicações de melhorias para Processo de Manufatura (PM 1) e Desenvolvimento de Produto (PM 3).

Ao percebermos o excessivo número de indicações de melhorias voltadas para ações fundamentais ao andamento de uma empresa, notamos que boa parte delas ainda está em um nível de desenvolvimento principiante. Visto isso, é pertinente mencionar que as empresas que se encontram neste nível necessitam, antes do ingresso no mercado externo, posicionamento no mercado interno e estratégias de manutenção no mercado.

A necessidade de investimentos em áreas como flexibilidade, inovação, qualidade na produção e promoção de marcas trazidos por Skoludova e Kozena (2015) se mostram como imprescindíveis à criação de vantagens competitivas. Essas dimensões se mostram presentes nas indicações de demandas propostas pelo PEIEX, no entanto o indicativo de melhorias a essas áreas não se mostrou efetivo no que diz respeito ao quantitativo de empresas que conseguiu efetivas a atividade de exportação proposta no programa.

Nesse sentido, a partir da análise dos dados do PEIEX juntamente à literatura pertinente, fica clara a necessidade de acompanhamento da implantação das melhorias junto às entidades parceiras bem como do oferecimento de atividades visando o desenvolvimento das empresas. O comprometimento do empresário também se mostra essencial ao desenvolvimento das capacidades da empresa e, portanto, faz-se preciso o direcionamento dos esforços para o cumprimento das atividades direcionadas pelos Técnicos Extensionistas, cabendo ao Núcleo Operacional e a ApexBrasil a intermediação.

Como indicação está, tanto para o PEIEX quanto para as entidades parceiras, a difusão da cultura exportadora para que as empresas passem a enxergar os reais ganhos potenciais com o processo de exportação. Para isso é necessário que o empresário conheça as etapas necessárias ao processo de exportação, saiba quais os benefícios que a empresa terá com o processo, o que é preciso fazer para sua empresa iniciar o processo de exportação, mas, primordialmente é imprescindível que o empresário seja capaz de responder por si só a pergunta "por quê eu devo exportar?".

Como sugestão para estudos futuros está o estabelecimento de parâmetros para mensuração da competitividade. Levando em consideração a relevância das MPE, a criação de indicadores de desempenho da capacidade competitiva vem como um fator favorável à adoção de estratégias de internacionalização eficientes e análise de programas de fomento distintos e seus impactos. 
Espera-se que as discussões trazidas nesse estudo contribuam para o direcionamento de políticas públicas que se mostrem eficazes ao desenvolvimento das MPE e à exportação, promovendo assim ganhos econômicos e sociais.

Assim, conclui-se que esses programas se tornam mais efetivos no caso de serem aplicados em ambientes de inovação controlados e continuados voltados para atenderem demandas do mercado internacional com o suporte de universidades e institutos de pesquisa juntamente com empresas. Dessa forma tornam-se capazes de produzirem esses produtos depois de vencidas as barreiras tecnológicas de produção liberando assim para competição no mercado internacional após atingir o estágio de maturidade tecnológica necessário.

\section{Referências}

Abebe, M. A. Angriawan, A. (2014). Organizational and competitive influences of exploration and exploitation activities in small firms. Journal of Business Research 67 339-345. DOI: 10.1016/j.jbusres.2013.01.015

Bell, J. (1995). The internationalization of small computer software firms. European journal of marketing. https://doi.org/10.1108/03090569510097556

Boehe, D. M., Larentis, F., De Toni, D., \& Mattia, A. Á. (2011). Papel das relações interorganizacionais e da capacidade de inovação na propensão para exportar. REAd. Revista Eletrônica de Administração (Porto Alegre), 17(1), 86-116. https://doi.org/10.1590/S141323112011000100004

Bonaccorsi, A. (1992). On the relationship between firm size and export intensity. Journal of international business studies, 23(4), 605-635. https://doi.org/10.1057/palgrave.jibs.8490280

Calof, J. L. (1994). The relationship between firm size and export behavior revisited. Journal of International Business Studies, 25(2), 367-387. https://doi.org/10.1057/palgrave.jibs.8490205

Coutinho, C. P. (2014). Metodologia de investigação em ciências sociais e humanas. Leya.

Coviello, N. (2015). Re-thinking research on born globals. Journal of International Business Studies, 46(1), 17-26.

Coviello, N., \& Munro, H. (1997). Network relationships and the internationalisation process of small software firms. International business review, 6(4), 361-386. 
Czinkota, M. R. (1986). International trade and business in the late 1980s: An integrated US perspective. Journal of International Business Studies, 17(1), 127-134.

Da Rosa, F. M., de Mello, R. C., Ferreira, V. A. de C., (2018). The phenomenon of internationalization and scale-up enterprises: A systematic literature review. Internext, 01 August 2018, Vol.13(2), pp.71-85 https://doi.org/10.18568/1980-4865.13271-85

Dal-Soto, F., Alves, J. N., \& Bulé, A. E. (2014). Análise do Processo de Internacionalização do Modelo de Uppsala: caminhos para as empresas Brasileiras. Revista Estudo \& Debate, 21(1).

De Melo, M. A. \& Leone, R. J. G. (2015). Alinhamento entre as Estratégias Competitivas e a Gestão de Custos: um Estudo em Pequenas Empresas Industriais do Setor de Transformação. BBR Brazilian business review (Portuguese ed.), 01 January 2015, Vol.12(5), pp.83-104

De Oliveira, J., Terence, A. C. F., \& Escrivão Filho, E. (2010). Planejamento estratégico e operacional na pequena empresa: impactos da formalização no desempenho e diferenças setoriais. Revista Gestão Organizacional, 3(1), 119-133.

De Oliveira, M. C. S. F., Scherer, F. L., Hahn, I. S., Carpes, A. de M., Dos Santos, M. B., Piveta, M. N. (2018). Drivers of international performance of Brazilian technology-based firms. Internext, 01 January 2018, Vol.13(1), pp.32-49. https://doi.org/10.18568/1989-4865.13132-49

Deluiz, N. (2004). A globalização econômica e os desafios à formação profissional. Boletim técnico do Senac, 30(3), 73-79. FLICK, Uwe. Triangulation in qualitative research. A companion to qualitative research, v. 3, p. 178-183, 2004. https://doi.org/10.18568/1989-4865.13132-49

Dzikowski, P. (2018) A bibliometric Analysis of born global firms. Journal of business research, April 2018, Vol.85, pp.281-294 DOI: 10.1016/j.jbusres.2017.12.054

Domingues, L. M., Muritiba, P. M., Muritiba, S. N., (2016). Good corporate governance in micro and small business takes internationalisation? Contextus (Fortaleza), 01 December 2016, Vol.14(3), pp. $53-78$

Etemad, H. (2017). Towards an emerging evolutionary life-cycle theory of internationalized entrepreneurial firms: from born globals to borderless firms?. Journal of International 
Entrepreneurship, 15(2), 111-120. DOl:10.1057/jibs.2014.62

Francischini, A. S. N. , Furtado, J., Garcia, R. (2015). Tecnologia e trajetórias de internacionalização precoce: análise de casos na indústria brasileira. Gestão \& Produção, 01 June 2015, Vol.22(2), pp.267-279 : https://doi.org/10.24023/FutureJournal/2175-5825/2021.v13i1.437

Gil, A. C. (2002). Como elaborar projetos de pesquisa (Vol. 4, p. 175). São Paulo: Atlas.

Gohr, C. F., Silva, Y. L. T. V. S. (2015). Managing the relationship between strategic resources and competitive priorities through the resource-based view. Revista produção online, 01 June 2015, Vol.15(2), pp.734-757 DOI:10.1057/jibs.2014.62

Günther, H. (2006). Pesquisa qualitativa versus pesquisa quantitativa: esta é a questão?. Psicologia: teoria e pesquisa, 22(2), 201-209.

Haahti, A., Madupu, V., Yavas, U., \& Babakus, E. (2005). Cooperative strategy, knowledge intensity and export performance of small and medium sized enterprises. Journal of world business, 40(2), 124-138.

Heinzmann, L. M., \& Machado, D. D. P. N. (2014). Cultura Organizacional e estágios de internacionalização: um estudo em quatro empresas do segmento de metal-mecânico-elétrico brasileiro. BBR-Brazilian Business Review, 11(2), 35-66.

Kao, J. Nação Inovadora (2008). QulityMark, Rio de janeiro - Brasil.

Kraus, P. G. (2000). Modelo de internacionalização de empresas produtoras exportadoras brasileiras.

Knight, G. A., \& Cavusgil, S. T. (2004). Innovation, organizational capabilities, and the born-global firm. Journal of international business studies, 35(2), 124-141. doi:10.1057/palgrave.jibs.8400071

Knight, G. A., \& Liesch, P. W. (2016). Internationalization: From incremental to born global. Journal of World Business, 51(1), 93-102.DOI: 10.1016/j.jwb.2015.08.011

Kotler, P. (2000). Administração de marketing: a edição do novo milênio.

Kraus, P. G. (2006). O Processo de Internacionalização das Empresas: o caso brasileiro. Revista de Negócios, Blumenau, 11(2), 25-47. http://dx.doi.org/10.7867/1980-4431.2006v11n2p\%25p 
Leone, R. J. G., \& Guerra, N. M. D. C. P. (2011). Pequenas e Médias Empresas: contribuições para a discussão sobre por que e como medir o seu tamanho. RAUnP-ISSN 1984-4204-Digital Object Identifier (DOI): http://dx. doi. org/10.21714/raunp., 4(1), 67-83.

Mcdowell, William C ; Harris, Michael L; Geho, Patrick R (2016) Longevity in small business: The effect of maturity on strategic focus and business performance Journal of business research, May 2016, Vol.69(5), pp.1904-1908. DOI: 10.1016/j.jbusres.2015.10.077

Mozzato, A. R. \& Grzybovski, D. (2018). Global mindset: Premise for developing competitive advantage in international markets. Internext, 01 January, Vol. 13(1), pp. 77-89. DOI: 10.18568/19804865.13177-89

Nascimento, A. L. S., Armani, A., Santos, J. L., Hansen, P. B., (2017). Competências Organizacionais no Contexto dos Relacionamentos Interorganizacionais. Revista de Administração IMED, 01 December 2017, Vol.7(2), pp.71-97

Organização para cooperação econômica e desenvolvimento - OCDE. (2005). Manual de Oslo: Diretrizes para coleta e interpretação de dados sobre inovação [manual]. 2a versão.

Organização para cooperação econômica e desenvolvimento - OCDE. (2020). Relatórios econômicos Brasil 2020.

Palmer-Rubin, Brain. (2016) Interest organizations and distributive politics: small-business subsides in Mexico. World development, August, vol 84, pp. 97-117.

Peiris, I. K., Akoorie, M. E., \& Sinha, P. (2012). International entrepreneurship: A critical analysis of studies in the past two decades and future directions for research. Journal of International Entrepreneurship, 10(4), 279-324.

Porter, M. (2004). Estrategia competitiva. Elsevier Brasil.

Tunes, R. \& Monteiro, P. R. R. (2017). Conhecimento em Gestão, Vantagem Competitiva e Performance Empresarial: Proposição e Teste de um Modelo Fundamentado na "Resource Advantage Theory" em MPEs. Revista brasileira de marketing, 01 August 2017, Vol.16(3), pp.298-316 
Rennie, M. W. (1993). Born global. The McKinsey Quarterly, (4), 45-53.DOI: 10.4236/ti.2015.61006

Ruzzier, M., Antončič, B., \& Konečnik, M. (2006). The resource-based approach to the internationalisation of SMEs: Differences in resource bundles between internationalised and non-internationalised companies. Zagreb International Review of Economics \& Business, 9(2), 95-116.

Sarfati, G. (2013). Estágios de desenvolvimento econômico e políticas públicas de empreendedorismo e de micro, pequenas e médias empresas (MPMESSs) em perspectiva comparada: os casos do Brasil, do Canadá, do Chile, da Irlanda e da Itália (Vol. 47-1), Revista de Administração Pública - Rio de Janeiro, pp. 25-48. https://doi.org/10.1590/S0034-76122013000100002

Schumpeter, J. (1988). A teoria do desenvolvimento econômico. São Paulo: Nova Cultural.

Serviço Brasileiro de Apoio às Micro e Pequenas Empresas (SEBRAE). (2014) A Evolução das Microempresas e Empresas de Pequeno Porte 2009 a 2012 Brasil. Série Estudos e Pesquisas.

Serviço Brasileiro de Apoio às Micro e Pequenas Empresas (SEBRAE). (2014 a) Participação das Micro e Pequenas Empresas na Economia Brasileira. Julho.

Serviço Brasileiro de Apoio às Micro e Pequenas Empresas (SEBRAE). (2015). Micro e pequenas empresas geram 27\% do PIB do Brasil.

Serviço Brasileiro de Apoio às Micro e Pequenas Empresas (SEBRAE). (2015a). Participação das micro e pequenas empresas na economia brasileira. Brasília: Sebrae, 2015a.

Serviço Brasileiro de Apoio às Micro e Pequenas Empresas (SEBRAE). (2020). As micro e pequenas empresas na exportação brasileira. Brasil: 2009-2016, Recuperado em: 25 julho de 2020. < http://www.bibliotecas.sebrae.com.br/chronus/ARQUIVOS_CHRONUS/bds/bds.nsf/54159d7c e6a88bfbbf9d32a0a9d58f3b/\$File/7796.pdf >.

Simoes, V. C., Rocha, A. D., Mello, R. D., \& Carneiro, J. (2015). Black Swans or an Emerging Type of Firm? The Case of Borderless Firms. The Future of Global Organizing (Progress in International Business Research, 10, 179-200.

Skoludova, J., \& Kozena, M. (2015). Identification of the Tools and Methods of Selected Factors of 
Organization's Competitiveness in the Czech Republic. Procedia Economics and Finance, 26, 609-615.

Sousa, C. M., \& Novello, S. (2014). The influence of distributor support and price adaptation on the export performance of small and medium-sized enterprises. International Small Business Journal, 32(4), 359-385. https://doi.org/10.1177/0266242612466876

Tidd, J., \& Bessant, J. (2015). Gestão da inovação-5. Bookman Editora.

Toyne, B., \& Nigh, D. (1997). Foundations of an emerging paradigm. Toyne, Brian/Nigh, Douglas, 3-26.

Vahlne, J. E., \& Johanson, J. (2017). From internationalization to evolution: The Uppsala model at 40 years. Journal of International Business Studies, 48(9), 1087-1102.

Vasconcelos, F. C., \& Cyrino, Á. B. (2000). Vantagem competitiva: os modelos teóricos atuais e a convergência entre estratégia e teoria organizacional. Revista de Administração de empresas, 40(4), 20-37. https://doi.org/10.1590/\$0034-75902000000400003

Zhao, Ming ; Dong, Ciwei ; Cheng, T.C.E. (2018) Quality disclosure strategies for small business entrepises in a competitive marketplace. European journal of operational research, 01 October 2018, Vol.270(1), pp.218-229 https://doi.org/10.1016/j.ejor.2018.03.030 